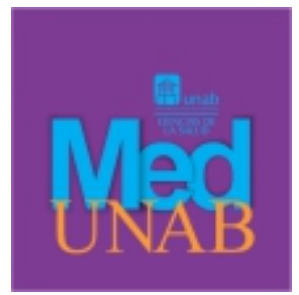

REVISTA DE LA FACULTAD

DE CIENCIAS DE LA SALUD

Vol. 22(2):213-227, agosto - noviembre 2019

Revisión de tema

\title{
Actualización en sepsis y choque séptico en adultos
}

Update on Sepsis and Septic Shock in Adults

Atualização sobre sepse e choque séptico em adultos

Marlon Adrián Laguado-Nieto, MD., Esp. ${ }^{1} \mathbb{0}$, Amaury Alexis Amaris-Vergara, MD., Esp. ${ }^{2}{ }^{\oplus}$, Jhon Edgar Vargas-Ordóñez, MD. ${ }^{3} \oplus$, Jully Andrea Rangel-Vera, $M D .{ }^{4}{ }^{\circ}$, Susan Juliett García-León, MD. ${ }^{5}$, Katherine Tatiana Centeno-Hurtado, $M D .{ }^{6}$

1. Médico, Especialista en Medicina Interna, Clínica FOSCAL Internacional, Floridablanca, Santander, Colombia. Fellow de Medicina Crítica y Cuidado Intensivo del adulto, Universidad Autónoma de Bucaramanga, Bucaramanga, Santander, Colombia. Bucaramanga, Santander, Colombia. Clínica FOSCAL Internacional, Floridablanca, Santander, Colombia.

2. Médico, Especialista en Medicina Interna, Clínica Los Comuneros, Bucaramanga, Santander, Colombia.

3. Médico, Especialista en Medicina Interna, Clínica Los Comuneros, Bucaramanga, Santander, Colombia.

4. Médico, Toronto, Canadá.

5. Médico, Hospital Internacional de Colombia, Floridablanca, Santander, Colombia.

6. Médico, Residente de Medicina Interna, Universidad Industrial de Santander, Bucaramanga,Santander, Colombia.

Correspondencia: Marlon Adrián Laguado Nieto, Médico Especialista en Medicina Interna. Universidad de Santander. Carrera 28 \#33-43. Dirección postal: 680002 Bucaramanga. Colombia. E-mail: adriannieto_007@hotmail.com

\section{INFORMACIÓN ARTÍCULO}

Artículo recibido: 22 de agosto de 2018

Artículo aceptado: 28 de julio de 2019

DOI: https://doi.org/10.29375/01237047.3345

\section{RESUMEN}

Introducción. La sepsis se define como una disfunción multiorgánica secundaria a un proceso infeccioso que puede progresar a choque séptico con aumento en el riesgo de mortalidad. Recientes actualizaciones de Sepsis nos permiten realizar un diagnóstico precoz y abordaje terapéutico oportuno con disminución de la morbimortalidad a corto y mediano plazo.

División de los temas tratados. Se realizó una revisión bibliográfica no sistemática en bases de datos y bibliotecas electrónicas (PubMed, Cochrane, Lilacs) que incluyó artículos en inglés y español desde 2005 hasta 2018 que contuvieran los tópicos de interés. Se realizó una descripción detallada de la definición de sepsis y choque 


\section{Cómo citar.}

Laguado-Nieto MA, Amaris-Vergara

AA, Vargas-Ordóñez JE, Rangel-Vera JA, García-León SJ, Centeno-Hurtado KT.

Actualización en sepsis y choque séptico en adultos. MedUNAB.

2019;20(2):213-227.

doi:10.29375/01237047.3345 séptico, clasificación, fisiopatología, diagnóstico, monitorización hemodinámica y tratamiento.

Conclusiones. La sepsis es una urgencia médica con una alta tasa de mortalidad que requiere de un diagnóstico oportuno, el cual se puede realizar por medio del puntaje del SOFA (Sepsis Related Organ Failure Assessment), y el inicio rápido de tratamiento antibiótico empírico y reanimación guiada por metas, ya que son medidas que han demostrado disminuir los desenlaces fatales.

Palabras clave:

Sepsis; Choque séptico; Microcirculación; Insuficiencia multiorgánica; Manejo de la enfermedad.

\section{ABSTRACT}

Introduction. Sepsis is defined as a multiple organ dysfunction secondary to an infectious process that can progress to septic shock, increasing the risk of mortality. Recent findings pertaining to Sepsis allow us to perform early diagnoses and timely therapeutic treatments with short and medium-term reductions in mortality.

Division of Covered Topics. Non-systematic literary research was conducted in databases and digital libraries (PubMed, Cochrane, Lilacs), including articles in English and Spanish from 2005 to 2018 that included the topics of interest. A detailed description was made of the definition of sepsis and septic shock, their classification, physiopathology, diagnosis, hemodynamic monitoring and treatment.

Conclusions. Sepsis is a medical emergency with a high mortality rate that requires a timely diagnosis. The diagnosis can be performed with the Sepsis Related Organ Failure Assessment (SOFA) score, and quickly beginning empirical antibiotic treatment and reanimation guided by goals, as these measures have shown a reduction in fatal outcomes.

Keywords: Sepsis; Septic shock; Microcirculation; Multi-organ failure; Disease management.

\section{RESUMO}

Introdução. A sepse é definida como uma disfunção de múltiplos órgãos, secundária a um processo infeccioso que pode evoluir para choque séptico com risco aumentado de mortalidade. Atualizações recentes da sepse nos permitem fazer um diagnóstico precoce e uma abordagem terapêutica oportuna, com diminuição da morbidade e mortalidade a curto e médio prazo.

Divisão dos temas abordados. Foi realizada uma revisão bibliográfica não sistemática em bases de dados e bibliotecas eletrônicas (PubMed, Cochrane, Lilacs) incluindo artigos em inglês e espanhol de 2005 a 2018, que continham os tópicos de interesse. Foi realizada uma descrição detalhada da definição da sepse e choque séptico, classificação, fisiopatologia, diagnóstico, monitorização hemodinâmica e tratamento.

Conclusões. A sepse é uma emergência médica com uma alta taxa de mortalidade que requer um diagnóstico oportuno, que pode ser feito através do escore SOFA (Sepsis Related Organ Failure Assessment); o início rápido do tratamento antibiótico empírico e a ressuscitação guiada por metas, já que são medidas que demonstraram reduzir os resultados fatais.

Palavras-chave:

Sepse; Choque séptico; Microcirculação; Insuficiência multiorgânica; Gerenciamento clínico. 


\section{Introducción}

La sepsis es una entidad clínica definida como disfunción orgánica causada por una infección potencialmente fatal (1). La severidad de la sepsis puede variar desde un grado leve de disfunción hasta un compromiso circulatorio (choque séptico) con alta tasa de mortalidad. Este desenlace puede ser modificado si se realiza un diagnóstico temprano y si se llevan a cabo las medidas terapéuticas necesarias (2). Esta enfermedad es muy frecuente en el ámbito de urgencias, salas de hospitalización y cuidado crítico, donde la atención inicial juega un rol importante en la estabilización y sobrevida de los pacientes; por tal motivo, el objetivo principal en esta revisión no sistemática es la actualización en el manejo integral de los pacientes con sepsis y choque séptico, discutiendo las nuevas definiciones, abordaje diagnóstico y aspectos claves del tratamiento con líquidos, monitorización y el manejo antibiótico empírico.

\section{Definición y epidemiología}

El tercer consenso internacional de definición de sepsis y choque séptico publicado en el año 2016 introduce una nueva definición de sepsis, entendida como la "disfunción multiorgánica causada por una respuesta desregulada del huésped a una infección" (3), producida por una sobre activación (aumento de la inflamación) o supresión de la respuesta inmune (inmunosupresión). El nuevo concepto hace énfasis en la disfunción orgánica, eliminando el término "sepsis severa" empleado en guías anteriores $(3,4)$.

Anualmente, más de 18 millones de personas en el mundo sufren de sepsis, con una incidencia de 66 a 300 casos por 100,000 habitantes en países desarrollados.
La incidencia va en aumento debido al envejecimiento de la población ( $60 \%$ de los pacientes sépticos tienen edad mayor o igual a 65 años), a comorbilidades propias de la edad y al uso de tratamientos inmunosupresores con una mortalidad calculada entre el $27 \%$ y $36 \%$ (5). Por año se presentan alrededor de 5 millones de muertes a pesar de los avances en el entendimiento de la enfermedad, y la mortalidad promedio es mayor del $30 \%(5,6)$. Lograr las metas de reanimación en sepsis y choque séptico se ha asociado con descenso en los índices de mortalidad; la presión arterial media $(\mathrm{PAM}) \leq 65 \mathrm{mmHg}$ y la no respuesta a la reanimación con líquidos se ha asociado a un incremento en los desenlaces fatales. (7).

En Latinoamérica, existen pocos estudios acerca del comportamiento epidemiológico de la sepsis; sin embargo, en Colombia se realizó un estudio con 826 pacientes diagnosticados con sepsis, de los cuales el 51 $\%$ la desarrolló por infecciones adquiridas en la comunidad, el $44 \%$ en la UCI, y solo el $5 \%$ durante la estancia en hospitalización en salas generales; siendo las infecciones intra abdominales el diagnóstico más frecuente en un $18.6 \%$, seguida por neumonía adquirida en el hospital con $17 \%$, y la neumonía adquirida en comunidad en un $12.4 \%$. Las comorbilidades más frecuentes fueron la enfermedad pulmonar obstructiva crónica (EPOC), falla cardiaca y diabetes mellitus (DM) (6).

\section{Síndrome de respuesta inflamatoria sistémica (SRIS)}

Es un estado pro inflamatorio usualmente causado por infección; los criterios para síndrome de respuesta inflamatoria sistémica (Tabla 1) no son requeridos para el diagnóstico de sepsis, ya que entre el $10 \%$ y $12 \%$ de

Tabla 1. Criterios diagnósticos SRIS

\begin{tabular}{|c|c|}
\hline \multirow{4}{*}{$\begin{array}{c}\text { Definido } \\
\text { por la } \\
\text { presencia de } 2 \\
\text { o más signos }\end{array}$} & Temperatura $>38^{\circ} \mathrm{o}<36^{\circ}$. \\
\hline & Frecuencia cardiaca $>90 \mathrm{lpm} *$. \\
\hline & $\begin{array}{l}\text { Taquipnea, frecuencia respiratoria }>22 \mathrm{rpm}^{* *} \mathrm{o} \\
\text { hiperventilación }\left(\mathrm{PaCO} 2 * * *<32 \mathrm{mmHg} \mathrm{mH}^{* * *}\right) .\end{array}$ \\
\hline & $\begin{array}{l}\text { Leucocitos }>12,000 / \mathrm{mm}^{3} \mathrm{o}<4,000 / \mathrm{mm}^{3} \text { o más } \\
\text { de } 10 \% \text { neutrófilos inmaduros. }\end{array}$ \\
\hline
\end{tabular}

*lpm: latidos por minutos; ${ }^{* *}$ rpm: respiraciones por minuto; $* * * \mathrm{PaCO} 2$ : presión arterial Co2;

****mmHg: milímetros de mercurio

Fuente: Adaptado de Scott M. Defining and Diagnosing Sepsis. Emerg Med Clin N Am 35(2017) 1-9. 
los pacientes con sepsis en las unidades de cuidados intensivos (UCI) no cumplen con estos criterios; por tal motivo, estos parámetros por sí solos no son útiles para identificar la totalidad de los pacientes con sepsis (5). Asimismo, la pancreatitis, la isquemia, el trauma e injuria tisular, o el choque hemorrágico, pueden también causar SRIS (8).

\section{Choque séptico y falla multiorgánica}

Se configura con la presencia de los criterios de sepsis más la documentación de hipotensión persistente que requiere de soporte vasopresor para mantener una $\mathrm{PAM} \geq 65 \mathrm{mmHg}$ y un nivel de lactato sérico $\geq 2$ $\mathrm{mmol} / \mathrm{L}$, a pesar de manejar un volumen intravascular adecuado con cristaloides $(1,3,8)$. Actualmente, cuando mencionamos el término falla multiorgánica nos referimos al síndrome clínico que se caracteriza por disfunción de 2 o más órganos, o sistemas inducidos por sepsis. (1)

\section{Clasificación: escala SOFA (Sequential Organ Failure Assessment) y qSOFA (Quick SOFA)}

La escala SOFA es una herramienta que permite evaluar objetivamente la severidad de la disfunción orgánica (Tabla 2). En el contexto de un paciente con infección, el diagnóstico clínico de sepsis se realiza si se cumple con 2 o más criterios; cuanto más alto es el puntaje mayor probabilidad de muerte $(1,3,5)$.

Tabla 2. Escala SOFA

\begin{tabular}{|c|c|c|c|c|c|c|}
\hline Sistemas & Indicador & 0 & 1 & 2 & 3 & 4 \\
\hline Respiración & $\mathrm{PaO} 2 / \mathrm{FiO} 2$ & 400 & $<400$ & $<300$ & $<200^{*}$ & $<100^{*}$ \\
\hline Coagulación & $\begin{array}{c}\text { Plaquetas } \\
\text { x } 103 / \mathrm{ul}\end{array}$ & 150,000 & $<150.000$ & $<100,000$ & $<50,000$ & $<20,000$ \\
\hline Hígado & $\begin{array}{c}\text { Bilirrubinas } \\
\text { (mg/dl) }\end{array}$ & $<1.2$ & $1.2-1.9$ & $2.5-5.9$ & $6.0-11.9$ & $>12$ \\
\hline SCV & & $\begin{array}{c}\mathrm{PAM} \geq 70 \\
\mathrm{mmHg}\end{array}$ & $\begin{array}{c}\mathrm{PAM}<70 \\
\mathrm{mmHg}\end{array}$ & $\begin{array}{c}\text { Dopamina }< \\
5^{\mathrm{a}} \mathrm{o} \\
\text { dobutamina } \\
\text { a cualquier } \\
\text { dosis }\end{array}$ & $\begin{array}{c}\text { Dopamina } \\
5.1-15 \text { o } \\
\text { Epinefrina } \\
\leq 0.1 \text { o } \\
\text { Norepinefrina } \\
\leq 0.1\end{array}$ & $\begin{array}{c}\text { Dopamina }^{\mathrm{a}}> \\
15 \mathrm{o} \\
\text { Epinefrina }> \\
0.1 \mathrm{o} \\
\text { Norepinefrina } \\
>0.1\end{array}$ \\
\hline SNC & $\begin{array}{c}\text { Escala coma } \\
\text { Glasgow }\end{array}$ & 15 & $13-14$ & $10-12$ & $6-9$ & $<6$ \\
\hline \multirow[t]{2}{*}{ Renal } & $\begin{array}{c}\text { Creatinina } \\
\text { (mg/dl) }\end{array}$ & $<1.2$ & $1.2-1.9$ & $2.0-3.4$ & $3.5-4.9$ & $>5.0$ \\
\hline & $\begin{array}{c}\text { Gasto } \\
\text { urinario } \mathrm{ml} / \mathrm{d}\end{array}$ & & & & $<500$ & $<200$ \\
\hline
\end{tabular}

* Con soporte respiratorio; $\mathrm{PaO} 2$ (presión arterial de Oxigeno); FiO2 (Fracción inspirada de oxígeno; PAM (Presión arterial media).

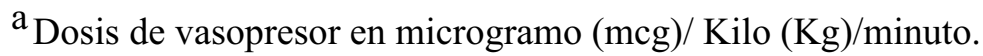

Fuente: Adaptado de Singer M et al. The Third International Consensus Definitions for Sepsis and Septic Shock (Sepsis -3). JAMA. 2016;315(8):801-810. 
Los cambios en la definición de sepsis tienen como objetivo identificar a los pacientes más enfermos, quienes son los que se benefician de una pronta intervención y en quienes se requiere del inicio oportuno de antibióticos de amplio espectro (5).

Existe una forma abreviada de la escala SOFA que no requiere variables de laboratorio, la cual se denominada qSOFA, esta incluye los siguientes criterios: a) frecuencia respiratoria $\geq 22 / \mathrm{min}$.; b) alteración del estado mental, escala de Glasgow < 15 y presión arterial sistólica (PAS) $\leq 100 \mathrm{mmHg}$ $(1,3)$

\section{Fisiopatología de la sepsis y del choque séptico}

La microcirculación comprende los elementos más pequeños del sistema circulatorio, una red de arteriolas, capilares y vénulas presentes en todos los órganos del cuerpo cuyo diámetro es menor de $150 \mu \mathrm{m}$ y se encarga de suplir los nutrientes y oxígeno necesarios para el metabolismo aerobio (9); la sepsis y el choque séptico afectan directamente la microcirculación. Esto ocurre como resultado del daño endotelial (fase preponderante en la fisiopatología del choque séptico) mediada de manera celular, principalmente por los leucocitos en el momento de interactuar con el endotelio y por mediadores inflamatorios, (prostaglandinas, especies reactivas de oxígeno o proteasas) los cuales lesionan el endotelio, produciendo aumento de la permeabilidad capilar y disminución del tono vascular por vasodilatación, lo que conduce a hipovolemia relativa, hipotensión, inadecuada perfusión a órganos, depresión miocárdica, choque y muerte $(10,11)$.

Además del deterioro en la perfusión tisular, también se evidencia una incapacidad de las células para extraer y utilizar adecuadamente el oxígeno. Estas alteraciones pueden persistir aun después de corregir las anormalidades hemodinámicas (10).

Un mecanismo importante en la disfunción microcirculatoria es el aumento de producción del Óxido Nítrico (NO) que puede explicar la poca respuesta a los agentes vasoactivos. En estados sépticos la isoenzima inducible de NO se activa por medio de citocinas y endotoxinas, generando gran cantidad de NO que desencadena vasodilatación arteriolar, empeorando la disfunción macro y microcirculatoria $(9,11,12)$.
Otro mecanismo de alteración en la perfusión tisular es la alteración en la coagulación durante la sepsis secundaria a disfunción endotelial. Las concentraciones de antitrombina se reducen por aumento de su degradación o alteración en la producción; la proteína $\mathrm{C}$, un anticoagulante endógeno, está alterada por disminución en la síntesis e incremento en su consumo. Adicionalmente, la sepsis se encuentra asociada a una deficiencia relativa del ADAMTS13 (un tipo de desintegrina y metaloproteasa con trombospondina tipo 1 no. 13), lo cual resulta en acúmulos de grandes multímeros de factor Von Willebrand, que facilitan adhesión plaquetaria al endotelio dañado. El factor tisular (FT) es el iniciador principal de la coagulación en sepsis; la actividad del inhibidor de la vía del FT asociado al factor VIIa se puede alterar en sepsis, y conlleva a la activación de coagulación intravascular diseminada (CID) posterior a exposición con los lipopolisacáridos de las bacterias (Figura 1) (13).

El deterioro en la oxigenación tisular es el punto final que explica la disfunción multiorgánica; la hipoxia tisular causa daño severo mitocondrial afectando la cadena respiratoria, lo que conlleva disminución de los niveles de Adenosin Trifosfato (ATP) y caída en el consumo de Oxígeno $(14,15)$.

\section{Diagnóstico}

El foco infeccioso puede no ser aparente al inicio debido a que el paciente puede referir variedad de síntomas inespecíficos (disnea, dolor abdominal, entre otros) con un amplio número de diagnósticos diferenciales. Resulta de vital importancia registrar en la historia clínica los antecedentes de infecciones recientes, exposición a antibióticos, cirugías y cultivos previos. En el examen físico se debe tener presente la presencia de rubor, calor, dolor, alteración en la funcionalidad, estado de conciencia, y presencia de dificultad respiratoria. Deben evaluarse cicatrices en busca de intervenciones quirúrgicas recientes, presencia de dispositivos médicos como catéteres, drenajes (nefrostomía, drenaje biliar), sonda vesical entre otros (16).

Se debe recolectar muestras de laboratorio y obtener cultivos previos al inicio de antibióticos, sin retrasar por ello su administración; se sugiere un retraso no mayor a 45 minutos entre la toma de cultivos y el inicio de la terapia antimicrobiana. Se deben recolectar al menos dos tomas de hemocultivos por vía percutánea (frascos de aerobios y anaerobios), y en caso de que el paciente 


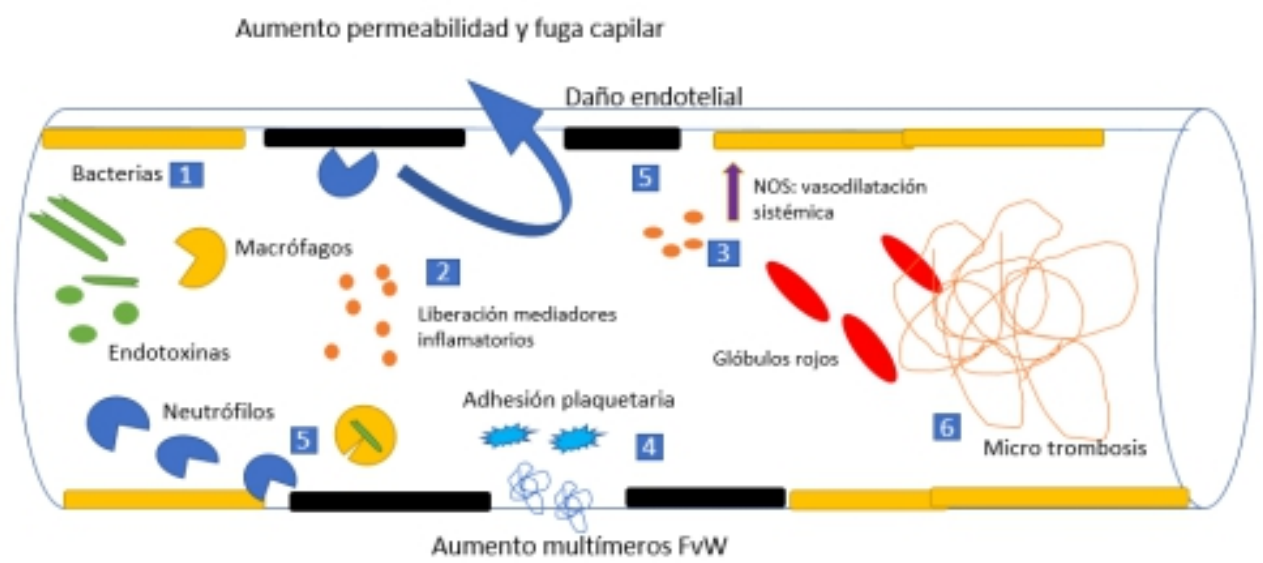

Foco infeccioso

Figura 1. Infección del torrente sanguíneo por bacterias, los cuales liberan endotoxinas e inician cascada de respuesta inflamatoria (1-2). En sepsis, la isoenzima iNOS se activa por la acción de citocinas y endotoxinas, lo que genera aumento del NO, desencadenando vasodilatación arteriolar (3). Se evidencia incremento en la adhesión plaquetaria, aumento en los polímeros de factor de von Willebrand por alteración en la proteasa ADAMTS 13 (4). Las células endoteliales se encuentran lesionadas, daño mediado principalmente por leucocitos; esto predispone al aumento de permeabilidad y fuga capilar, y asociado a la vasodilatación sistémica por el NO, hipotensión arterial e hipoperfusión tisular (5). La alteración en las proteínas anticoagulantes y el estado protrombótico de la sepsis origina formación de microtrombos a nivel intra vascular y puede desencadenar coagulación intravascular diseminada. Estos microtrombos alteran aún más la perfusión tisular en la microcirculación (6). En resumen, la vasodilatación arteriolar (que produce disminución presión arterial media y disminución perfusión tisular) y el aumento de permeabilidad por el daño endotelial que lleva a fuga capilar conduce a un estado de hipoperfusión tisular, disminución de aporte y consumo de oxígeno tisular, y esto lleva a disfunción mitocondrial, metabolismo anaerobio y disfunción orgánica.

Fuente: Elaborado por los autores.

tenga insertado algún dispositivo endovascular por más de 48 horas (ejemplo, un catéter venoso) se deberá tomar hemocultivos por cada uno de los lúmenes $(1,16)$.

\section{Biomarcadores en sepsis}

\section{Procalcitonina (PCT)}

Es un péptido cuya síntesis está regulada por el gen 1 calcitonina (CALC-1), su producción se activa en todos los tejidos en respuesta a una infección bacteriana mediada por la interleucina 6 (IL-6), factor de necrosis tumoral (FNT) e interleucina 1B (17). Se eleva en diversas situaciones clínicas como trauma, quemaduras severas, cirugía mayor (en especial posterior a bypass cardiopulmonar), estados post reanimación, pancreatitis aguda o hemorragia subaracnoidea; su presencia es altamente sensible para infección bacteriana con mejor rendimiento que la Proteína C reactiva y la IL-6 $(18,19)$.

Un punto de corte de $0.5 \mathrm{ng} / \mathrm{ml}$ tiene una sensibilidad de $72.6 \%$ y una especificidad del $69.5 \%$ para bacteriemia, aunque no se ha establecido un valor específico para el diagnóstico de sepsis (20).

Diversos trabajos han evaluado el inicio y suspensión de antibióticos basados en los niveles de PCT. Un estudio indica iniciar terapia antimicrobiana con concentración de PCT $>0.5 \mathrm{ng} / \mathrm{ml}$, con recomendación fuerte a favor de valores $>1 \mathrm{ng} / \mathrm{ml}$ y no se aconseja el uso de antibióticos con valores por debajo de $0.5 \mathrm{ng} / \mathrm{ml}$ (21).

Existen estudios que concluyen que la implementación de un protocolo antibiótico en pacientes críticos 
basados en los valores de PCT se asocia con una disminución en la duración del tratamiento sin un impacto significativo en el desenlace clínico final o en términos de costo efectividad (22). Adicionalmente, una revisión sistemática de Cochrane, en donde se incluyeron 10 ensayos con un total de 1215 participantes, concluyó que no hay suficiente evidencia para soportar el uso de antibióticos guiado por valores de PCT para disminuir mortalidad, duración de ventilación mecánica, reinfección o duración de tratamiento antibiótico en pacientes sépticos (23).

\section{Ácido Láctico}

La hiperlactatemia en sepsis se origina por tres procesos principales: en primer lugar, la hipoxia tisular que precipita el metabolismo anaerobio; en segundo lugar, por el incremento en la vía de glicolisis que excede la capacidad de la enzima piruvato deshidrogenasa para catalizar la conversión de piruvato a acetil coenzima $\mathrm{A}$, aumentando de este modo los niveles de lactato; $\mathrm{y}$, en tercer lugar, debido al aumento de la actividad de la bomba $\mathrm{Na} / \mathrm{K}$ ATPasa por estimulación beta adrenérgica (24).

Independientemente de su etiología, la hiperlactatemia en sepsis está relacionada de manera importante con un incremento en la tasa de mortalidad; por ejemplo, los pacientes hipertensos por sepsis sin hiperlactatemia tienen mejor pronóstico que los pacientes hipertensos con acidosis láctica $(24,25)$.

Los pacientes con una disminución inicial del lactato en 24 horas tienen mejores desenlaces que los pacientes con niveles en sangre persistentemente elevados (25). Se ha documentado que la normalización del lactato por debajo de $2 \mathrm{mmol} / \mathrm{L}$ durante la reanimación temprana en sepsis es el predictor más fuerte de sobrevida, seguido por una depuración del lactato del $50 \%$ dentro de las primeras 6 horas de presentación (26).

\section{Tratamiento}

Existen pilares básicos en el tratamiento como los líquidos endovenosos, los vasopresores y los antibióticos de amplio espectro, principalmente en los pacientes que están con choque séptico. Es crucial el inicio temprano del tratamiento debido a que cualquier dilación en el mismo puede precipitar la evolución hacia disfunción orgánica múltiple y muerte (27). En las últimas guías encontradas se documenta la reanimación por metas con el fin de disminuir la mortalidad asociada (Tabla 3).

Tabla 3. Objetivos de la Resucitación en Sepsis y Choque Séptico

\section{Dentro de la primera hora}

- Resucitación con cristaloides: Iniciar retos de volumen iniciales con bolos de hasta $30 \mathrm{cc} / \mathrm{kg}$.

a. Reducir la rata de infusión si hay aumento de la presión de cavidades cardíacas sin mejoría hemodinámica concurrente.
Dentro de las primeras 3 horas

- Medición sérica de lactato

- Iniciar antibioticoterapia de amplio espectro (preferiblemente dentro de la primera hora)

- Obtener al menos 2 hemocultivos previo al inicio del tratamiento si estos no implican un retraso mayor a $45 \mathrm{~min}$
Dentro de las primeras 6 horas

- PVC: 8-12 mm hg (>12 mmHg si VMI)

- PAM: $>65 \mathrm{mmHg}$

- $\mathrm{GU}>0.5 \mathrm{ml} / \mathrm{kg} / \mathrm{h}$

- $\mathrm{ScvO} 2>70 \%$

- $\mathrm{SvO} 2>65 \%$

- Aclaramiento del lactato a una velocidad aproximada de un 20 $\%$ cada 2 horas.

- Control de la fuente de infección si es posible.

$$
\begin{aligned}
& \text { Dentro de las primeras } \\
& 24 \text { horas } \\
& \text { - Glucosa }<180 \\
& \text { mg/dl } \\
& \text { - En pacientes } \\
& \text { ventilados: } \\
& \text { Presiones } \\
& \text { meseta }<30 \\
& \text { cmH2O } \\
& \text { - Hemoglobina }> \\
& 7 \text { mg/dl } \\
& \text { Plaquetas } \\
& >20.000 \text { si el } \\
& \text { paciente tiene } \\
& \text { alto riesgo de } \\
& \text { sangrado. }
\end{aligned}
$$

Abreviaturas: PVC: Presión venosa central. PAM: presión arterial media. Svo2: saturación venosa central.GU: gasto urinario. VMI: Ventilación mecánica invasiva.

Referencias: Rhodes A et al. Surviving Sepsis Campaign: International Guidelines for Management of Sepsis and Septic Shock 2016. Intensive Care Med (2017) 43:304-377.

Singer M, Deutschman CS, Seymour C, et al. The third international consensus definitions for sepsis and septic shock (SEPSIS-3). JAMA2016;315(8):801-10. 


\section{Líquidos endovenosos: guiado por metas}

La administración de líquidos es la medida de primera línea para pacientes con choque séptico, puesto que mejora el gasto cardiaco (GC), restaura la entrega de oxígeno y revierte la hipoxia tisular (28). No obstante, es necesario señalar que una reanimación agresiva puede resultar en sobrecarga de volumen y llevar a disfunción orgánica con incremento en la mortalidad (29).

La respuesta a los líquidos se advierte como un incremento en el volumen sistólico (VS) con la administración de estos. Según la curva de Frank Starling, el incremento en el llenado de los ventrículos (retorno venoso) aumenta la precarga; esto resulta en una elevación del GC hasta un límite superior en donde un incremento adicional no se ve reflejado en el VS y el GC, en cambio puede derivar en sobrecarga de volumen $(29,30)$; sumado a esto, la sepsis produce disfunción miocárdica $(29,31)$. El $50 \%$ de los pacientes con choque séptico tiene disfunción sistólica del ventrículo izquierdo, $62 \%$ disfunción diastólica del ventrículo izquierdo y el $31 \%$ puede tener disfunción ventricular derecha $(29,32)$. Un exceso en los líquidos administrados puede llevar a edema pulmonar, hipertensión pulmonar, empeoramiento de la función ventricular derecha y caída en el gasto cardiaco (29).

Existen parámetros para medir volemia y guiar la terapia con líquidos. Los parámetros estáticos como la presión venosa central (PVC) o la presión de oclusión arteria pulmonar (medida con catéter Swan Ganz), son índices hemodinámicos de presión o volumétricos que evalúan la precarga en un solo punto en el tiempo. La PVC (que mide la presión del volumen de sangre presente en la vena cava superior y, que, a su vez, es una medida estimada directa de la presión de la aurícula derecha y de la presión de fin de diástole del ventrículo derecho) recomendada es de 8-12 $\mathrm{mmHg}$ en pacientes con respiración espontánea y $12-15 \mathrm{mmHg}$ en paciente con ventilación mecánica (31).

Se ha debatido sobre el uso de la PVC como reflejo de la precarga, volumen intravascular total y como parámetro para evaluar la respuesta a los líquidos (33). Varios factores hacen de la PVC un método no fiable en la reanimación con líquidos, ya que puede verse afectada por cambios en la presión torácica, pericárdica e intraabdominal (34), lo cual deriva en un bajo valor predictivo reportado en algunas series de casos hasta en un $47 \%(31,34)$.
Existen datos que sugieren qué valores extremos de PVC pueden ayudar a guiar la terapia con líquidos $(\mathrm{PVC}<6-8 \mathrm{mmHg}$ y $>12-15 \mathrm{mmHg})$, sin ser útil en valores intermedios (34).

Los parámetros dinámicos son más sensibles que los estáticos; actualmente se considera el Gold standard para guiar la terapia hídrica dada la mejoría en su capacidad predictiva $(34,35)$.

El principio de los parámetros dinámicos es inducir un cambio en la precarga y observar el efecto resultante en el volumen sistólico o gasto cardíaco. Este cuantifica la variación del volumen sistólico inducida por la ventilación por presión positiva (36).

Durante la inspiración aumenta la presión intratorácica lo cual desencadena la disminución de la precarga del ventrículo derecho por dificultad del retorno venoso y, por tanto, reduce el volumen de eyección; por el contrario, la sangre contenida en la circulación pulmonar llega al ventrículo izquierdo aumentando su precarga y su volumen de eyección durante la fase inspiratoria. Al completar el tiempo de tránsito pulmonar se reduce el volumen sistólico del ventrículo derecho, lo cual condiciona una caída del llenado ventricular izquierdo y una reducción del volumen de eyección durante la fase de espiración; es decir, la ventilación mecánica produce cambios en el volumen sistólico incrementándose durante la inspiración y disminuyéndolo en espiración (37).

Cuando el ventrículo es muy dependiente de la precarga (porción ascendente de la curva de Frank Starling) la variación del volumen sistólico (VVS) es mayor, es decir, a mayor VVS mayor es la susceptibilidad a los cambios de precarga que lleva a una mejoría del gasto cardiaco con la administración de líquidos $(37,38)$. Una variación del volumen sistólico $\geq 10 \%$ o una variabilidad presión de pulso (VPP) $\geq 13 \%$ predicen respuesta al aporte de volumen con alta sensibilidad y especificidad $(37,38)$. Si una VVS es $<10 \%$ y el gasto cardiaco no responde al volumen, la hipotensión del paciente podrá ser manejada con soporte vasopresor y/o inotrópicos dependiendo de la función cardíaca (39).

Otros parámetros útiles en la predicción de la respuesta a fluidos (que se basan en el mismo principio fisiológico que la VVS) incluyen la variación de la velocidad del flujo aórtico evaluada mediante doppler esofágico, la variación del pico de velocidad o de la integral velocidad tiempo del flujo aórtico determinado por ecocardiograma y la variación de la vena cava superior e inferior (38). 
Como limitaciones para usar estos parámetros se encuentra la necesidad de ventilación mecánica invasiva en modos controlados, presencia de volumen corriente $>8 \mathrm{ml} / \mathrm{kg}$ y ausencia de arritmias cardiacas $(36,38)$.

\section{Líquidos endovenosos: ¿cuál elegir?}

Existen tres tipos de cristaloides: solución de dextrosa con o sin electrolitos, solución salina y las soluciones balanceadas (40). La solución salina $0.9 \%$ es el cristaloide más frecuentemente utilizado, contiene 154 $\mathrm{mmol} / \mathrm{L}$ de sodio y $154 \mathrm{mmol} / \mathrm{L}$ de cloro $(28,40)$. Se ha determinado que este cristaloide tiene efectos negativos en los riñones, en el equilibrio ácido base y homeostasis electrolítica (28). La hipercloremia puede conducir a disminución de flujo en arteria renal y disminuir filtración glomerular ya que el cloro a nivel de la mácula densa libera adenosina, la cual tiene efectos vasoconstrictores en la arteriola renal aferente (41).

De acuerdo con el abordaje fisicoquímico de Stewart, los cationes fuertes $\left(\mathrm{Na}^{+}, \mathrm{K}^{+}, \mathrm{Mg}^{2+}, \mathrm{Ca}^{2+}\right)$ predominan en relación con los aniones fuertes, produciendo una carga plasmática neta positiva de aproximadamente $42 \mathrm{mmol} / \mathrm{L}$, la cual se conoce como diferencia de iones fuertes (DIF) (42); esta carga plasmática positiva debe ser contrabalanceada con una carga igual negativa para mantener la neutralidad eléctrica (ley de electroneutralidad) (28). La carga de aniones se deriva de ácidos débiles no volátiles, principalmente albúmina y fosfato (28). La infusión de grandes cantidades de solución salina $0.9 \%$ produce acidosis metabólica hiperclorémica $(28,43,44)$. Al administrar esta solución el cloro plasmático aumenta, la carga neta positiva en plasma se reduce con el fin de mantener la neutralidad eléctrica, y al recuperar cargas positivas se aumentan las concentraciones de $\mathrm{H}+$ por su disociación del agua, disminuyendo el $\mathrm{pH} .(28,44)$.

Las soluciones balanceadas constituyen una mejor alternativa a la solución salina. Se considera balanceada a una solución con una DIF de 24 meq/L; esto se puede alcanzar si se remueven $24 \mathrm{meq} /$ litro de cloro de la solución salina $0.9 \%$, reemplazándolos con bicarbonato o aniones orgánicos como lactato o acetato, ya que es ligeramente más hipotónico que el líquido extracelular, acercándose más al $\mathrm{pH}$ plasmático; las soluciones balanceadas más usadas incluyen Lactato de Ringer, Acetato de Ringer y el Plasma-Lyte $(44,45)$. La administración excesiva de soluciones balanceadas puede producir alcalosis metabólica, hiperlactatemia e hipotonicidad. Sin embargo, resulta una opción más fisiológica; por tanto, pueden ser usados como cristaloides de elección (40).

Los coloides son moléculas con alto peso molecular, por lo tanto, no atraviesan las membranas capilares sanas. Pueden expandir el volumen intravascular efectivamente y mantener presión oncótica. La albúmina es un coloide derivado del plasma humano, los coloides semisintéticos son las gelatinas, dextranos y el Hidroxietil starches (HES); este último relacionado con falla renal y aumento en mortalidad $(43,44)$.

\section{Vasopresores}

Se recomienda el uso de norepinefrina como vasopresor de elección si persiste $\mathrm{PAM}<65 \mathrm{mmHg}$ a pesar de carga de líquidos $(30 \mathrm{ml} / \mathrm{kg})$, con el fin de mantener una presión arterial media $\geq 65 \mathrm{mmHg}$ $(46,47)$.

La dopamina no debe usarse como primera elección: la evidencia demuestra mayor mortalidad y aumento de incidencia de arritmias comparado con norepinefrina, ya que su uso está contemplado para el paciente con bradicardia $(46,47)$.

La epinefrina se utiliza como alternativa o en adición a la norepinefrina cuando esta no logra alcanzar metas de reanimación. Es el vasoactivo de primera línea en paro cardiaco y, del mismo modo que la dopamina, se recomienda para el uso en bradicardia sintomática que no responde a la atropina (47).

La vasopresina es un péptido hormonal que produce vasoconstricción por vía de los receptores $\mathrm{V} 1$, y es un coadyuvante en el tratamiento del choque séptico; no se recomienda su uso como único vasopresor (47) (Tabla 4).

Los inotrópicos se reservan para pacientes con disfunción miocárdica, bajo gasto cardiaco, aumento en las presiones de llenado, o signos de hipoperfusión tisular a pesar de restaurar volemia y alcanzar PAM en metas con líquidos y vasopresores (47)(Tabla 4).

\section{Tratamiento antibiótico}

El inicio apropiado y oportuno de la terapia antibiótica resulta fundamental para determinar sobrevida en pacientes con sepsis y choque séptico, ya que un tratamiento inicial inefectivo se ha visto relacionado 
Tabla 4. Agentes vasopresores e inotrópicos utilizados en el manejo del choque séptico

AGENTES VASOACTIVOS E INOTRÓPICOS

\begin{tabular}{|c|c|c|c|c|c|c|c|c|c|c|}
\hline \multirow[t]{2}{*}{ Nombre } & \multirow[t]{2}{*}{ Indicaciones } & \multirow[t]{2}{*}{$\begin{array}{l}\text { Actividad en Sepsis } \\
\text { y choque séptico }\end{array}$} & \multicolumn{6}{|c|}{ Actividad receptora } & \multirow[t]{2}{*}{ Dosis usual } & \multirow[t]{2}{*}{$\begin{array}{c}\text { Efectos } \\
\text { adversos }\end{array}$} \\
\hline & & & a1 & $B 1$ & $B 2$ & $D$ & $V 1-V 2$ & $\begin{array}{c}\text { PDE3 } \\
\text { antagonista }\end{array}$ & & \\
\hline Norepinefrina & $\begin{array}{l}\text { Choque (Séptico- } \\
\text { Cardiogénico) }\end{array}$ & $\begin{array}{l}\text { Vasoconstricción, } \\
\text { moviliza } \\
\text { sangre en el } \\
\text { sistema venoso, } \\
\text { soporte inotrópico }\end{array}$ & " & $\uparrow \uparrow \uparrow$ & $\uparrow \uparrow$ & 0 & 0 & 0 & $\begin{array}{c}0.02-0.3 \\
\mathrm{mcg} / \mathrm{kg} / \mathrm{min}\end{array}$ & $\begin{array}{l}\text { Taquiarrtimias, } \\
\text { apoptosis de } \\
\text { células } \\
\text { miocárdicas, } \\
\text { isquemia distal. }\end{array}$ \\
\hline Vasopresina & $\begin{array}{l}\text { Choque (Séptico, } \\
\text { vasopléjico) } \\
\text { +/-paro cardíaco }\end{array}$ & $\begin{array}{l}\text { Vasoconstricción de } \\
\text { la vasculatura } \\
\text { pulmonar y } \\
\text { sistémica, } \\
\text { coadyuvante }\end{array}$ & 0 & 0 & 0 & 0 & $\uparrow \uparrow \uparrow \uparrow \uparrow$ & 0 & $\begin{array}{l}0.01-0.04 \\
\mathrm{U} / \mathrm{min}\end{array}$ & $\begin{array}{l}\text { Taquiarritmias, } \\
\text { isquemia distal }\end{array}$ \\
\hline Adrenalina & $\begin{array}{l}\text { Choque (Séptico- } \\
\text { Cardiogénico), } \\
\text { paro cardíaco, } \\
\text { bradicardia } \\
\text { sintomática, } \\
\text { anafilaxia }\end{array}$ & $\begin{array}{l}\text { Vasoconstricción, } \\
\text { efecto B1 a bajas } \\
\text { dosis, a }>0.1 \\
\mathrm{mcg} / \mathrm{kg} / \mathrm{min}\end{array}$ & $\uparrow \uparrow \uparrow \uparrow \uparrow$ & $\uparrow \uparrow \uparrow \uparrow$ & $\uparrow \uparrow \uparrow$ & 0 & 0 & 0 & $\begin{array}{c}0.01-0.2 \\
\mathrm{mcg} / \mathrm{kg} / \mathrm{min}\end{array}$ & $\begin{array}{c}\text { Arritmias } \\
\text { ventriculares, } \\
\text { Hipertensión } \\
\text { severa, ECV, } \\
\text { isquemia distal } \\
\text { acidemia } \\
\text { metabólica, } \\
\text { acidosis láctica }\end{array}$ \\
\hline Dobutamina & $\begin{array}{l}\text { Choque séptico- } \\
\text { cardiogénico, } \\
\text { cardiomiopatía, } \\
\text { falla cardíaca } \\
\text { descompensada, } \\
\text { estados de bajo GC }\end{array}$ & $\begin{array}{l}\text { Inotrópico- } \\
\text { inodiladator }\end{array}$ & $\uparrow$ & $\uparrow \uparrow \uparrow \uparrow \uparrow$ & $\uparrow \uparrow$ & 0 & 0 & 0 & $\begin{array}{c}5-15 \\
\mathrm{mcg} / \mathrm{kg} / \mathrm{min}\end{array}$ & $\begin{array}{l}\text { Arritmias } \\
\text { ventriculares, } \\
\text { isquemia } \\
\text { cardíaca, } \\
\text { hipotensión por } \\
\text { el fecto B2 }\end{array}$ \\
\hline Dopamina & $\begin{array}{l}\text { Bradicardia } \\
\text { sintomàtica, } \\
\text { choque (séptico, } \\
\text { cardiogénico) }\end{array}$ & $\begin{array}{l}\text { Inotropía a dosis } \\
\text { intermedias, } \\
\text { vasodiltación por lo } \\
\text { receptores DA a } \\
\text { dosis ajas y } \\
\text { vasocontricción a } \\
\text { altas dosis }\end{array}$ & $\uparrow \uparrow \uparrow$ & $\uparrow \uparrow \uparrow \uparrow \uparrow$ & $\uparrow \uparrow$ & $\uparrow \uparrow \uparrow \uparrow \uparrow$ & 0 & 0 & $\begin{array}{c}2-20 \\
\mathrm{mcg} / \mathrm{kg} / \mathrm{min}\end{array}$ & $\begin{array}{l}\text { Taquiarritmias, } \\
\text { isquemia } \\
\text { cardiaca, } \\
\text { hipertensión } \\
\text { severa }\end{array}$ \\
\hline Fenilefrina & $\begin{array}{c}\text { Hipotensión } \\
\text { aguda (Vagal, } \\
\text { medicamentosa), } \\
\text { estenosis aórtica } \\
\text { con hipertensión } \\
\text { cardiomiopatía } \\
\text { hipertrófica con } \\
\text { obstrucción del } \\
\text { tracto de salida }\end{array}$ & $\begin{array}{l}\text { Vasonstricción, } \\
\text { coadyuvante }\end{array}$ & $\uparrow \uparrow \uparrow \uparrow \uparrow$ & 0 & 0 & 0 & 0 & 0 & $\begin{array}{c}100-180 \\
\mathrm{mcg} / \mathrm{min} \\
\text { inicialmente, } \\
40-60 \\
\text { mcg/min para } \\
\text { mantenimiento, } \\
\text { bolos de } 50- \\
200 \mathrm{mcg} \mathrm{c} / 20 \\
\text { min }\end{array}$ & $\begin{array}{l}\text { Empeoramiento } \\
\text { de la función } \\
\text { cardíaca, } \\
\text { barorreflejo, } \\
\text { bradicardia }\end{array}$ \\
\hline Milrinone & $\begin{array}{l}\text { Estados con bajo } \\
\text { GC, refractarios al } \\
\text { manejo con } \\
\text { dobutaminay/c } \\
\text { pacientes } \\
\text { crónicamente } \\
\text { betabloqueados, } \\
\text { pacientes con falla } \\
\text { derecha con } \\
\text { hipertensión } \\
\text { pulmonar }\end{array}$ & $\begin{array}{l}\text { Inodilatación: } \\
\text { disminuye } \\
\text { resistencias } \\
\text { pulmonares }\end{array}$ & & $\begin{array}{l}\text { Equivalente: } \\
\quad \uparrow \uparrow \uparrow \uparrow \uparrow\end{array}$ & $\begin{array}{c}\text { Equivalente: } \\
\qquad \uparrow \uparrow \uparrow \uparrow\end{array}$ & & & 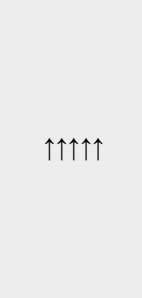 & $\begin{array}{c}0.25-0.75 \\
\mathrm{mcg} / \mathrm{kg} / \mathrm{min}\end{array}$ & $\begin{array}{l}\text { Efectos } \\
\text { vasodilatadores } \\
\text { marcados, } \\
\text { torsade de } \\
\text { pointes, otras } \\
\text { arritimias } \\
\text { ventriculares }\end{array}$ \\
\hline
\end{tabular}

\section{Convenciones: $\uparrow \uparrow \uparrow \uparrow \uparrow: 100 \%$}

Fuente: adaptado de Stratton L, Berlin DA, Arbo JE. Vasopressors and Inotropes in Sepsis. Emerg Med Clin N Am 35 (2017) 75-9 
con desenlaces adversos $(49,50)$. Su inicio en la primera hora luego de la instauración del choque séptico está asociado con una sobrevida del $79.9 \%$ al alta hospitalaria (51). La prevalencia de un tratamiento antibiótico inicial inadecuado es del $10 \%$ al $40 \%$, impactando negativamente en la sobrevida hasta 5 veces en pacientes con choque séptico $(48,52)$.

La elección de la terapia antimicrobiana depende del sitio de la infección, los patógenos prevalentes en la comunidad y el hospital, el patrón de resistencia, el estado inmune del paciente, las comorbilidades, diagnóstico de falla hepática y/o renal, presencia de dispositivos invasivos (catéter venoso central, sonda vesical) y factores de riesgo para gérmenes resistentes (estancia hospitalaria prolongada, uso antibiótico reciente, colonización previa, infección por gérmenes multiresistentes) (51,53); por lo anterior, se deben establecer esquemas de manejo empírico inicial, de acuerdo con el sitio de infección y en casos de sitio no identificado.

Es necesario señalar que en pacientes con choque séptico la concentración sérica de los antibióticos se puede afectar debido a varios factores:

- Aumento del aclaramiento renal: la vasodilatación, el aumento del gasto cardiaco, los líquidos endovenosos y el soporte vasopresor pueden incrementar el flujo de sangre al riñón, aumentando no solo la depuración de creatinina sino también el aclaramiento de antibióticos hidrofílicos (como los betalactámicos), disminuyendo su concentración en sangre (54-56).

- La hipoalbuminemia aumenta la cantidad de moléculas no unidas a proteínas disponibles para ser filtradas y depuradas (54-56). Además, la cantidad de antibiótico no unida a proteínas se acumula en un tercer espacio, produciendo disminución de su concentración sérica y tisular; antibióticos como la oxacilina, ceftriaxona, ertapenem y daptomicina (57), poseen una alta fijación a la albúmina.

- Aumento del volumen de distribución: debido al daño endotelial y a la fuga capilar existe una extravasación de líquidos hacia el espacio intersticial (generando un tercer espacio); esto, asociado con la vasodilatación, produce hipotensión, razón por la cual debe administrarse un gran volumen de cristaloides durante la fase de reanimación. Lo anterior conlleva a un aumento en el volumen de distribución, siendo los antibióticos hidrofílicos (betalactámicos, glucopéptidos, aminoglicósidos, linezolid) los que más se afectan, ya que se disminuye su concentración $(54,55)$.
- La disminución en la perfusión tisular como consecuencia del choque séptico disminuye la concentración de los antibióticos en el sitio de la infección(56).

Debido a lo anterior, es importante la familiarización con conceptos farmacocinéticos y farmacodinámicos de los antibióticos con el fin de alcanzar las concentraciones en sangre ideales para erradicar la infección:

a) Antibióticos tiempo dependientes - Tiempo $>$ Concentración inhibitoria mínima (T>CIM): penicilinas, cefalosporinas, carbapenémicos, linezolid. Se recomienda lograr una máxima duración de exposición, la cual se consigue con una administración frecuente o infusión prolongada $(56,57)$; es así como la piperacilina tazobactam en dosis de 4.5 gr IV cada 8 horas o 3.375 gramos cada 6 horas está indicada para infecciones severas (51).

Algunos autores recomiendan después de la dosis inicial una infusión extendida de los medicamentos por varias horas, más que la administración estándar de 30 minutos (51); esto genera $\mathrm{T}>\mathrm{CIM}$ $100 \%$ para patógenos sensibles, en comparación con la administración intermitente (52).

b) Antibióticos concentración dependiente máxima sobre CIM (Cmax/CIM): se recomienda administración de altas dosis con el fin de maximizar su concentración y optimizar actividad bactericida $(56,57)$; se incluyen aminoglicósidos, metronidazol o daptomicina.

c) Antibióticos concentración dependiente con tiempo dependencia (Área bajo la curva: CIM): relación del área bajo la curva concentración tiempo durante un periodo de 24 horas a la CIM del patógeno; se busca maximizar la cantidad de antibiótico en sangre, lo cual se logra administrando una alta dosis total diaria $(56,57)$; incluyen fluoroquinolonas, glucopéptidos, aminoglucósidos, tigeciclina. La vancomicina debe ajustarse para obtener niveles séricos entre $15-20 \mathrm{mg} / \mathrm{L}$; esto se logra con una dosis de carga de $25-30 \mathrm{mg} / \mathrm{kg}$ (dosis de carga de 1 gramo no logrará niveles terapéuticos) (51).

\section{Evaluación de la microcirculación}

Durante la reanimación, además de monitorizar la depuración del lactato, se debe monitorizar la saturación venosa central de oxígeno $\left(\mathrm{ScvO}_{2}\right)$, que se obtiene de la aurícula derecha por medio de un catéter 
venoso central; esta tiene una correlación adecuada con la saturación venosa mixta de Oxígeno $\left(\mathrm{SvO}_{2}\right)$, la cual es obtenida de la arteria pulmonar, siendo ambas, buenos indicadores del transporte de $\mathrm{O}_{2}\left(\mathrm{DO}_{2}\right)(38)$. La $\mathrm{Scvo}_{2}$ representa el balance entre entrega y consumo de $\mathrm{O}_{2}$ (46). Un valor $<70 \%$ (en disminución del gasto cardiaco) indica DO2 inadecuado a los tejidos, un aumento en la extracción a nivel celular (por aumento en necesidades metabólicas) o una combinación de ambos $(31,38)$. Valores muy elevados de $\mathrm{ScvO} 2$ (mayor o igual 90\%), sugiere pobre utilización de O2, disoxia tisular, shunt en microcirculación y se asocia con alta mortalidad $(31,38)$.

En estados de hipoxia tisular se incrementa no solo el ácido láctico y aniones, también hay incremento del dióxido de carbono (CO2) (38). Para medir esto se utiliza el gradiente arteriovenoso de $\mathrm{CO} 2$ (valor normal 2-6 mmHg); valores elevados pueden indicar una inadecuada perfusión tisular (se puede detectar antes del aumento de lactato y puede aparecer con valores de $\mathrm{svcO} 2$ normal $)(31,38,58)$.

\section{Soporte transfusional}

Se recomienda transfusión de glóbulos rojos cuando la hemoglobina (HB) se encuentre $<7 \mathrm{~g} / \mathrm{dl}$ en ausencia de isquemia miocárdica, hipoxemia severa o sangrado agudo (51). Se puede mantener niveles de HB entre 7-9 $\mathrm{gr} / \mathrm{dl}$. Salvo que tengan indicaciones médicas que la amerite, la eritropoyetina no debe ser usada para tratar la anemia en estos pacientes. El plasma fresco congelado estará indicado en caso de coagulopatía asociado con sangrados o previo procedimiento invasivo (51).

\section{Soporte ventilatorio invasivo}

En caso de requerimiento de protección de la vía aérea e inicio de ventilación mecánica (pacientes con deterioro del estado conciencia), volumen corriente de $6 \mathrm{ml} / \mathrm{kg}$ disminuye mortalidad en pacientes que desarrollan síndrome de dificultad respiratoria del adulto (SDRA), se recomienda presiones meseta de hasta $30 \mathrm{cmH} 20$ y usar moderada presión positiva al final de la espiración (PEEP por sus siglas en ingles); posición prona en caso de SDRA severo inducido por sepsis $(27,51)$.

\section{Manejo hiperglucemia}

La hiperglucemia es inducida por hormonas de estrés (cortisol, hormona del crecimiento, glucagón, catecolaminas) y por citoquinas proinflamatorias, que producen gluconeogénesis y resistencia periférica a la insulina. Varios estudios asocian la hiperglucemia con un incremento en mortalidad (59).

Hacer un control estricto de la glucemia (80-110 $\mathrm{mg} / \mathrm{dl}$ ) puede aumentar la mortalidad por aumento en los episodios de hipoglucemia; por tal motivo, las guías recomiendan mantener una glucemia $\leq 180$ $\mathrm{mg} / \mathrm{dl}$ e iniciar insulina en presencia de valores mayores a $180 \mathrm{mg} / \mathrm{dl}(51,59)$.

\section{Corticoides}

En pacientes en estado crítico, existe una alteración en el eje hipotálamo hipófisis adrenal $(46,59)$. Se indica en caso de persistencia de hipotensión arterial que no esté respondiendo a líquidos y al soporte vasopresor Hidrocortisona $200 \mathrm{mg}$ IV día; no debe usarse en pacientes sépticos para prevenir el desarrollo de choque (1,51).

\section{Conclusiones}

La sepsis es la disfunción multiorgánica secundaria a un proceso infeccioso, con alta incidencia y mortalidad si progresa al estado de choque séptico. Se debe realizar una identificación oportuna de los pacientes con sepsis por medio del puntaje de la escala SOFA, tomar cultivos, buscar focos de infección e iniciar de manera rápida y apropiada el tratamiento antibiótico empírico con el fin de poder controlar la infección y evitar desenlaces fatales; además, en los pacientes con inestabilidad hemodinámica se deberá iniciar un manejo de reanimación integral guiado por metas con líquidos endovenosos, monitorizando por diversos métodos la respuesta al aporte de estos líquidos para evitar sobrecarga de volumen, soporte vasopresor y, en caso de requerirlo, soporte ventilatorio invasivo. E1 personal médico y asistencial de los servicios de urgencias, hospitalización y cuidado intensivo deberán tener claridad en los procesos diagnósticos y de tratamiento, y adherirse a las guías de manejo de esta entidad para poder impactar de forma positiva la sobrevida de los pacientes con sepsis y choque séptico.

\section{Conflicto de intereses}

Los autores declaran no tener conflicto de interés.

\section{Referencias}

1. Arsanios DM, Barragán AF, Garzón DA, Millán FC, Pinzón J, Ramos Isaza E, et al. Actualización en sepsis y choque séptico: nuevas definiciones y evaluación clínica. Acta Colomb Cuid Intensivo. 
[Online]. 2017;17(3):158-183. Disponible en: https://es.scribd.com/document/353841539/Actual izacio-n-en-sepsis-y-choque-se-ptico-nuevasdefiniciones-y-evaluacio-n-cli-nica

2. Perner A, Holst L, Haase N, Hjortrup P, Møller M. Common Sense Aproach to Managing Sepsis. Crit Care Clin. [Online]. 2018;34:127-138. Disponible en : https://www.mendeley.com/catalogue/ common-sense-approach-managing-sepsis/

3. Singer M, Deutschman C, Seymour C, ShankarHari M, Annane D, Bauer M, et al. The third international consensus definitions for sepsis and septic shock (sepsis-3). JAMA, 2016;35(8):801810. Disponible en :https://www.mendeley.com/ catalogue/third-international-consensusdefinitions-sepsis-septic-shock-sepsis3/

4. Marshall J. Sepsis Definitions: a Work in Progress.Crit Care Clin. 2018;34(1):1-14. Disponible en: https:// w w . criticalcare.theclinics.com/article/S07490704(17)30069-6/abstract

5. Keeley A, Hine P, Nsutebu E. The recognition and management of sepsis and septic shock: a guide for no intensivists. Postgrad Med J. 2017;1104(93):626-634. Disponible en: https://pmj.bmj.com/content/93/1104/626.long

6. Ortíz G, Dueñas C, Rodríguez F, Barrera L, de La Rosa G, Dennis R, et al. Epimediology of sepsis in Colombian Intensive care units. Biomédica: revista del Instituto Nacional de Salud. $2014 ; 34(1): 40-7$. Disponible en: https://www.researchgate.net/publication/271344 480_Epidemiologia_de_la_sepsis_en_unidades_ de cuidado intensivo en Colombia

7. Uvizl R, Adamus M, Cerny V, Dusek L, Jarkovsky J, Sramek V, et al. Patient survival, predictive factors and disease course of severe sepsis in Czech intensive care units: A multicentra, retrospective, observational study. Biomedical Papers. 2016;160(2): 287-297. Disponible en: https://www.ncbi.nlm.nih.gov/pubmed/26526190

8. Scott M. Defining and Diagnosing Sepsis Emergency Medicine Clinics of North America. $2017 ; 35(1): 1-9$. Disponible en: https://www.elsevier.com/books/severe-sepsiscare-in-the-emergency-department-an-issue-ofemergency-medicine-clinics-of-northamerica/perkins-jr/978-0-323-49646-9

9. Charlton M, Sims M, Coats T, Thompson JP. The Microcirculation and its measurement in sepsis. J Intensive Care Med. 2017;18(3):221-227. D i s p o i b l e e n : https://www.ncbi.nlm.nih.gov/pmc/articles/PMC 5665123/

10. Lipinska-Gediga M. Sepsis and septic shock -is a microcirculation a main player? Anaesthesiology Intensive Therapy. 2016;48(4):261-265. D is p on ib 1 e en: ht p s:// www.ncbi.nlm.nih.gov/pubmed/27660252

11. Russell J, Rush B, Boyd J. Pathophysiology of Septic Shock.Crit Care Clin. 2018;34(1):43-61. D i s p o n i b 1 e e n : https://www.criticalcare.theclinics.com/article/S0 749-0704(17)30070-2/fulltext

12. Pool R, Gomez H, Kellum JA. Mechanism of Organ Dysfunction in sepsis.Crit Care Clin. $2018 ; 34(1): 63-8$. Disponible en: https://www.criticalcare.theclinics.com/article/S0 749-0704(17)30068-4/fulltext

13. Opal SM, van der Poll T. Endothelial barrier dysfunction in septic shock. Journal of Internal Medicine. 2015;277(3):277-293. Disponible en: https://onlinelibrary.wiley.com/doi/full/10.1111/j oim. 12331

14. Angus DC, van der Poll T. Severe Sepsis and septic Shock. The New England journal of medicine. 2013;369(9):840-851. Disponible en: https://www.nejm.org/doi/full/10.1056/NEJMra1 208623

15. Chen C, Deng M, Sun Q, Loughran P, Billiar TR, Scott MJ. Lipopolysaccharide stimulates p62dependent autophagy-like aggregate clearance in hepatocytes. Biomed Res Int. 2014; 2014: 267350. doi:10.1155/2014/267350

16. Oliver Z, Perkins J. Source Identification and Source Control. Emerg Med Clin North Am. 2017 Feb;35(1):43-58. doi: 10.1016/j.emc.2016.08.005

17. Samsudin I, Vasikaran SD. Clinical Utility and Measurement of Procalcitonin. Clin Biochem Rev. 2017 Apr;38(2):59-68.

18. Bréchot N, Hékimian G, Chastre J, Luyt C. Procalcitonin to guide antibiotic therapy in the ICU.Int J Antimicrob Agents. 2015 Dec;46 Suppl 1: S19-24. doi: 10.1016/j.ijantimicag.2015.10.012.

19. van Engelen et al. Biomarkers in Sepsis. Crit Care Clin. 2018 Jan;34(1):139-152. doi: 10.1016/j.ccc.2017.08.010

20. Tsalik E, Jaggers L, Glickman S, Langley R, Van Velkinburgh J, Park L, et al. Discriminative Value of Inflammatory Biomarkers for Suspected Sepsis. J Emerg Med. 2012 Jul;43(1):97-106. doi: 10.1016/j.jemermed.2011.05.072

21. Bouadma L, Luyt C, Tubach F, Cracco C, Alvarez A, Schwebel C, et al. Use of procalcitonin to reduce patients' exposure to antibiotics in intensive care units (PRORATA trial): a multicentre randomised controlled trial. Lancet. 2010 Feb 6;375(9713):463-74. doi: 10.1016/S0140-6736(09)61879-1

22. Hohn et al. Procalcitonin-guided algorithm to 
reduce length of antibiotic therapy in patients with severe sepsis and septic shock. BMC Infect Dis. 2013 Apr 1; 13:158. doi: 10.1186/1471-2334-13158.

23. Andriolo BNG, Andriolo RB, Salomão R, Atallah Ï. Effectiveness and safety of procalcitonin evaluation for reducing mortality in adults with sepsis, severe sepsis or septic shock. Cochrane Database Syst Rev. 2017 Jan 18;1:CD010959. doi: 10.1002/14651858.CD010959.pub2.

24. Suetrong B, Walley KR. Lactic Acidosis in Sepsis: It's Not All Anaerobic. Implications for Diagnosis and Management. Chest. 2016 Jan;149(1):252-61. doi: 10.1378/chest.15-1703.

25. Fan S, Miller N, Lee J, Remick D. Diagnosis sepsis-The Role of Laboratory Medicine. Clin Chim Acta. 2016 Sep 1; 460:203-10. doi: 10.1016/j.cca.2016.07.002

26. Reddy AJ, Lam SW, Bauer SR, Guzman JA. Lactic acidosis: Clinical implications and management strategies. Cleve Clin J Med. 2015 Sep;82(9):61524. doi: 10.3949/ccjm.82a.14098.

27. Rello J, Valenzuela Sanchez F, Ruiz Rodriguez M, Moyano S. Sepsis: a review of advances in Management. Adv Ther. 2017 Nov;34(11):23932411. doi: 10.1007/s12325-017-0622-8

28. Corrêa TD, Cavalcanti AB, Assunção MS. Balanced crystalloids for septic shock resuscitation. Rev Bras Ter Intensiva. 2016 OctDec; 28(4): 463-471.doi: 10.5935/0103507X.20160079

29. Mujherjee V, Brosnahan SB, Bakker J. How to use Fluid Responsiveness in Sepsis. In: JL Vincent (Ed). Annual Uptodate in Intensive Care and Emergency Medicine 2017. Springer, Cham 69-80.

30. Greenwood JC, Orloski CJ. End Points of sepsis Resuscitation. Emerg Med Clin North Am. 2017 $\mathrm{F} \mathrm{e} \mathrm{b} ; 35(1): 93-107 . \quad \mathrm{d} \mathrm{o} \mathrm{i}$ : 10.1016/j.emc.2016.09.001.

31. Fenton KE, Parker M. Cardiac Function and dysfunction in sepsis. Clin Chest Med. 2016 J u n ; 3 7 ( 2 ) : 2 $89-98$ d o i : 10.1016/j.ccm.2016.01.014.

32. Henning D, Shapiro N. Goal Directed Resuscitation in septic shock: A critical Analysis. Clin Chest Med. 2016 Jun;37(2):231-9. doi: 10.1016/j.ccm.2016.01.016

33. De Backer D, Vincent JL. Should we measure the central venous pressure to guide fluid management? Ten answers to 10 questions.Crit Care. 2018 Feb 23;22(1):43. doi: 10.1186/s13054018-1959-3.

34. Chapalain X, Gargadennec T, Huet O. Fluid Balance during septic Shock:It's time to optimize. In: JL Vincent (Ed). Annual Uptodate in Intensive Care and Emergency Medicine 2017. Springer
International Publishing AG 2017.

35. Guerin L, Monnet X, Teboul JL. Monitoring volume and fluid responsiveness: From static to dynamic indicators. Best Pract Res Clin Anaesthesiol. 2013 Jun;27(2):177-85. doi: 10.1016/j.bpa.2013.06.002.

36. Sabatier C, Monge I, Maynar J, Ochagavia A. Valoración de la precarga y la respuesta cardiovascular al aporte de volumen. Med Intensiva. $2012 ; 36(1): 45-55$. doi: 10.1016/j.medin.2011.04.005

37. Ochagavía A, Baigorri F, Mesquida J, Ayuela J, Ferrándiz A, García X, et al. Monitorización Hemodinámica en el paciente crítico. Recomendaciones del Grupo de trabajo de Cuidados Intensivos Cardiológicos y RCP de la Sociedad Española de Medicina Intensiva, Crítica y Unidades Coronarias. Med Intensiva. 2014 A p r ; 3 8 ( 3$): 154-69$ d o i : 10.1016/j.medin.2013.10.006.

38. Davinson D, Basu RK, Goldstein SL, Chawla LS. Fluid Management in Adults and Children: core curriculum 2014. Am J Kidney Dis. $20144 ; 63(4): 7000-712$. https://doi.org/10.1053/j.ajkd.2013.10.044

39. Arshed S, Pinsky M. Applied Physiology of Fluid Resuscitation in critical illnes. Crit Care Clin. 2018 A p r ; 3 4 ( 2): 267-277. d o i : 10.1016/j.ccc.2017.12.010

40. Chang R, Holcomb JB. Choice of fluid therapy in the initial management of sepsis, severe sepsis, and septic shock. Shock. 2016 Jul;46(1):17-26. doi: 10.1097/SHK.0000000000000577.

41. Morgan TJ. Clinical review: The meaning of acidbase abnormalities in the intensive care unit-efects of fluid administration. Crit Care. 2005; 9(2): 204-211.doi: 10.1186/cc2946

42. McGuire MD, Heung M. Fluid as a drug: Balancing resuscitation and Fluid Overload in the Intensive Care Setting. Adv Chronic Kidney Dis. 2016 M a y;23 (3): $152-9$. d o i : 10.1053/j.ackd.2016.02.006.

43. Semler MW, Rice TW. Sepsis Resuscitation Fluid Choice and Dose. Clin Chest Med. 2016 J u n ; 37 ( 2 ): $241-50 . \quad$ d o i : 10.1016/j.ccm.2016.01.007.

44. Corrêa TD, Rocha LL, Pessoa CM, Silva E, de Assuncao MS. Fluid therapy for septic shock resuscitation: which fluid should be used? Einstein (Sao Paulo). 2015 Jul-Sep;13(3):462-8. doi: 10.1590/S1679-45082015RW3273.

45. Armstrong BA, Betzold RD, May AK. Sepsis and Septic Shock Strategies. Surg Clin North Am. 2017 D e c ; 97 ( 6 ) : $1339-1379$. d o i : 10.1016/j.suc.2017.07.003

46. Stratton L, Berlin DA, Arbo JE. Vasopressors and 
Inotropes in Sepsis.Emerg Med Clin North Am. 2017 F e b; 35 ( 1): $75-91$. d o i : 10.1016/j.emc.2016.09.005.

47. Allinson MG, Heil EL, Hayes BD. Appropriate Antibiotic Therapy. Emerg Med Clin N Am. 20177 ; $35(1): 25-42$. https://doi.org/10.1016/j.emc.2016.08.003.

48. Sharma S, Kumar A. Antimicrobial Management of sepsis and septic shock. Clin Chest Med. 2008 D e c ; 29 ( 4 ): 677-87, ix . d o i : 10.1016/j.ccm.2008.06.004.

49. Funk D, Kumar A. Antimicrobial Therapy for Life threatening infections: Speed is Life. Crit Care C1in. 2011 Jan;27(1):53-76. doi : 10.1016/j.ccc.2010.09.008.

50. Rhodes A, Evans L, Alhazzani W, Levy M, Antonelli M, Ferrer R, et al. Survivin Sepsis Campaing: International Guidelines for Management of Sepsis an Septic Shock 2016. Intensive Care Med. 2017 Mar;43(3):304-377. doi: 10.1007/s00134-017-4683-6.

51. Vazquez Grande G, Kumar A. Optimizing antimicrobial therapy of sepsis and septic shock: Focus on Antibiotic Combination Therapy. Seminars in Respiratory and Critical Semin Respir Crit Care Med. 2015 Feb;36(1):154-66. doi: 10.1055/s-0034-1398742.

52. Richter D, Heininger A, Brenner T, Hochreiter M, Bernhard M, Briegel J, et al. Bacterial sepsis. Diagnostics and calculated antibiotic therapy. Anaesthesist. 2017 Oct;66(10):737-761. doi: 10.1007/s00101-017-0363-8.

53. Roberts J, Abdul-Aziz M, Lipman J, Mouton J,
Vinks A, Felton T, et al. Individualised antibiotic dosing for patients who are critically ill: challenges and potential solutions. Lancet Infect Dis. 2014 Jun;14(6):498-509. doi: 10.1016/S14733099(14)70036-2.

54. Osthoff M, Siegemund M, Balestra G, Abdul Aziz $\mathrm{MH}$, Roberts JA. Prolonged administration od Blactamic antibiotics a comprehensive review and critical appraisal. Swiss Med Wkly. 2016 Oct 10;146:w14368. doi: 10.4414/smw.2016.14368.

55. Abdul-Aziz MH, Dulhunty JM, Bellomo R, Lipman J, Roberts JA. Continuous beta-lactam infusion in critically ill patients: the clinical evidence. Ann Intensive Care. 2012 Aug 16;2(1):37. doi: 10.1186/2110-5820-2-37.

56. Molina F, Gonzalez M, Guerra A, Muñoz F, Mendoza R. Farmacocinética y farmacodinamia de los antibióticos en el paciente críticamente enfermo. Primera parte. Acta Colomb de Cuid Intensivo. 2016;16(2)95-101.

57. Simpson N, Lamontagne F, Hari-Shankar M. Septic shock resuscitation in the first hour. Curr Opin Crit Care. 2017 Dec;23(6):561-566. doi: 10.1097/MCC.0000000000000460.

58. Ingels $\mathrm{C}$, Gunst J, Van den Berghe G. Endocrine and metabolic alterations in Sepsis and implications for treatment. Crit Care Clin. 2018 Jan;34(1):81-96. doi: 10.1016/j.ccc.2017.08.006 